\title{
Ontogeny of long-term, nonassociative memory in the rat
}

\author{
RICK RICHARDSON and BYRON A. CAMPBELL \\ Princeton University, Princeton, New Jersey
}

\begin{abstract}
The primary purpose of the present study was to assess the ontogeny of nonassociative learning and memory in 16-, 30-, and 75-day-old rats. In each of three experiments, habituation of the orienting response to a novel auditory stimulus was measured. The orienting response is an unconditioned reaction elicited by innocuous environmental stimulation that habituates with repeated stimulus presentations. Both an autonomic component (heart-rate deceleration) and a behavioral index (head jerk) of the orienting response were recorded in this study. Although no age differences in rate of habituation (i.e., rate of nonassociative learning) were found (see Experiment 1), a marked effect of age on retention of habituation was observed. Preweanling rats retained habituation of the orienting response to an auditory stimulus for less than $4 \mathrm{~h}$ (Experiment 2), whereas adult animals exhibited no forgetting even after a 1-week interval (Experiment 3). These results are discussed in terms of (1) demonstrations of age-related differences in associative memory, (2) the persistence of nonassociative memories in adults, and (3) the significance of the orienting response as a measure of retention of nonassociative learning.
\end{abstract}

During the last two decades, an increasing amount of research has been conducted on the development of associative memory in both humans and animals. These studies have provided overwhelming evidence that retention improves as the subject matures. Studies with human infants clearly demonstrate a dramatic improvement in memory performance during development. Using a conjugate mobile reinforcement procedure, Rovee-Collier and her colleagues have shown, for example, that 2-monthold infants remember for 1 day (Greco, Rovee-Collier, Hayne, Griesler, \& Earley, 1986), 3-month-olds remember for 8 days (Sullivan, Rovee-Collier, \& Tynes, 1979), and 6-month-olds remember for 14 days (Hill, Borovsky, \& Rovee-Collier, 1988). Similar findings in the developing rat are numerous. B. A. Campbell and E. H. Campbell (1962), for example, found that 18-day-old rats showed substantial forgetting of a fear memory, assessed by a spatial avoidance task, after only 7 days, whereas 100 day-old rats showed nearly perfect memory even after a 42-day interval. Comparable age differences in retention in the developing rat have been found with a wide variety of conditioning procedures (e.g., B. A. Campbell \& Spear, 1972; Miller \& Spear, 1989; Spear, Miller, \& Jaglio, 1990).

In contrast to the extensive research on the development of associative memory, considerably less effort has been expended on the development of nonassociative mem-

This research was supported by National Institute of Mental Health Grant MH01562 to Byron A. Campbell. The authors would like to thank Robert Schumm for his assistance with Experiment 2, and Harlene Hayne for her helpful comments on earlier versions of this paper. Reprint requests should be addressed to Byron A. Campbell, Department of Psychology, Princeton University, Princeton, NJ 08544-1010. ory in animals. Although the habituation paradigm has been used extensively to study memory in both human infants (e.g., Cohen \& Gelber, 1975) and adult invertebrates (e.g., Carew, Pinsker, \& Kandel, 1972), it has been used very infrequently in laboratory mammals. There are at least two studies, however, that examined age differences in retention of habituation in the rat. In one study, habituation of the forelimb withdrawal response to shock was examined (B. A. Campbell \& Stehouwer, 1980); in the other, habituation of a simple exploratory behavior (nose poking) was examined (Parsons, Fagan, \& Spear, 1973). In both cases, significant age differences in retention of habituation were reported; however, age differences in rate of learning were also seen in both studies, making conclusions regarding developmental differences in retention per se somewhat tenuous.

The ontogeny of long-term, nonassociative memory is of considerable contemporary theoretical interest for at least two reasons. The first has to do with the recent emphasis on the sequential emergence of learning capacity from the simple to the complex. Rudy and his colleagues have been the most active current proponents of this analysis (for review, see Rudy, Vogt, \& Hyson, 1984). In one series of experiments, for example, they found that although rat pups 9 days of age and older were able to exhibit a simple form of nonassociative learning, attenuation of neophobia, it was not until 12 days of age that they were able to exhibit a simple form of associative learning, conditioned taste aversion. Furthermore, a slight delay between taste and illness disrupted the formation of this aversion in animals 12 days old, but not in animals 15 days of age. The results of these experiments were taken as evidence that the ability to exhibit more complicated forms of learning emerges sequentially during the 
animal's development. If this line of reasoning is extended to include memory processes as well as learning ability, then long-term memory for nonassociative learning should emerge considerably earlier in development than should long-term memory for associative learning.

In addition, examination of the development of nonassociative memory is of current theoretical interest because of the widespread use of nonassociative procedures in research on the neurobiology of learning. Kandel and his colleagues (e.g. , Pinsker, Kupferman, Castellucci, \& Kandel, 1970; see also Bailey, Castellucci, Koester, \& Chen, 1983) and Thompson and his coworkers (e.g., Groves \& Thompson, 1970) have made extensive use of nonassociative procedures to study the neurobiological bases of learning. Some of the most exciting recent work in this area has involved a developmental analysis of habituation, sensitization, and dishabituation in aplysia (Marcus, Nolen, Rankin, \& Carew, 1988; Rankin \& Carew, 1988, 1989). Our knowledge concerning the development of nonassociative memory, however, lags far behind our knowledge of the development of nonassociative learning.

The orienting response (OR) is elicited by novel stimuli of low to moderate intensity, and it is a multicomponent reaction consisting of central, autonomic, and behavioral responses. The orienting response is generally assumed to reflect attention to a specific environmental stimulus and an early stage of sensory information processing. According to Sokolov (1963), when presentation of a novel stimulus elicits an OR, a trace or model of the stimulus is formed in the nervous system. Through repeated prese1tations of the stimulus, the neuronal model becomes an increasingly more accurate representation of external stimulation. Subsequent sensory input is compared with the neuronal model. If there is not a match between external stimulation and the model, the OR is again elicited and more or different attributes of the stimulus are encoded. If a match occurs, however, the OR is not elicited and habituation is observed. This type of comparator model of habituation is the basis for more general current theoretical formulations, such as Wagner's (1981) SOP model.

The OR was selected for the present research because it can serve as a prototypical model for the acquisition of environmentally relevant sensory information that occurs during normal development. The underlying assumption is that the developing organism acquires a great deal of information about its habitat through a passive, nonassociative process. During the course of ontogeny, the developing animal is exposed to an enormous variety of auditory, visual, and olfactory stimuli. Trees rustle, twigs crackle, shadows move, and odors waft. All of these could elicit the OR, but only a few are likely to signal negative or positive consequences. Habituation of the $O R$ to those stimuli that are unpredictive of future events is obviously essential given that the reactions comprising the OR are costly in terms of energy and time.

\section{EXPERIMENT 1}

The primary purpose of Experiment 1 was to describe the ontogeny of nonassociative learning and memory in 16-, 30-, and 75-day-old rats, using habituation and retention of habituation of the orienting response to an auditory stimulus as the measure. In the rat, a novel auditory stimulus elicits a dramatic decrease in heart rate (e.g., Richardson, Siegel, \& B. A. Campbell, 1988a) and a sudden jerk of the head to one side (Siegel, Sananes, Gaddy, \& B. A. Campbell, 1987). Both of these responses are considered characteristic components of the OR to auditory stimuli. Nonassociative learning was assessed by comparing the rate of habituation of the OR to 10 successive presentations of an auditory stimulus at the three ages. With the stimulus parameters used in this study, complete habituation should be observed well within this number of trials. Retention of nonassociative learning was measured by presenting the same auditory stimulus following a $24-h$ retention interval. If habituation to the auditory stimulus is retained, animals should not exhibit an OR when it is presented again after this interval. If, however, the animals have forgotten the previous presentations of the auditory stimulus after a 24-h interval, then they should exhibit comparable cardiac and behavioral responses to the auditory stimulus during each of the two series of exposures.

\section{Method}

Subjects. Thirty experimentally naive Sprague-Dawley-derived rats born in the Psychology Department's vivarium at Princeton University were used as subjects. Separate groups of animals were either 16, 30, or approximately 75 days of age on the first day of testing (all $n \mathrm{~s}=10$ ). Birth was counted as Day 0. On Day 2, all litters were culled to 8 animals each. No more than 2 animals from any single litter were included in the same group. Animals of both sexes were used at 16 days of age, but only males were used at the two older ages. The 16-day-olds were housed in $48 \times 25.5 \times 20.5 \mathrm{~cm}$ polycarbonate rodent cages with their dam and littermates. The older subjects were singly housed in $24.5 \times 17.5 \times 17 \mathrm{~cm}$ hanging wire mesh cages. All animals had ad-lib access to food and water and were maintained on a 16:8-h light:dark cycle (lights on at 7:30 a.m.). All testing was done during the light portion of the cycle.

Apparatus. Testing was performed in a clear Plexiglas chamber $(30 \times 17.5 \times 46 \mathrm{~cm})$. This chamber was located inside a singlewall Industrial Acoustics sound-attenuating chamber (IAC). Dim illumination was provided by a small incandescent nightlight located on one side of the IAC. A small window in the door of the IAC permitted observation of the animal.

Sensory stimulus. An 80-dB (as measured on Scale C of a Simpson Sound Level Meter) $1600-\mathrm{Hz}$ pulsating tone was used in Experiment 1 . This tone was generated by a Coulbourn Instruments precision signal generator, amplified by a Realistic audio amplifier (Model SA-10) and presented by a 4-in. speaker (Jensen) located inside the IAC. The tone was $10 \mathrm{sec}$ in duration and was pulsed at a rate of $2 \mathrm{sec}$ (rise/fall time $=250 \mathrm{msec}$ ).

Heart-rate recording procedure. Slightly different procedures were used to record heart rate in the different-aged subjects. To record heart rate in the 16- and 30-day-old subjects, two transcutaneous stainless steel electrodes ( $25 \mathrm{ga}$, approximately $1.5 \mathrm{~cm}$ in length) 
shaped like safety pins were used. These electrodes were acutely implanted just prior to test (this took 10-15 sec). One electrode was placed ventrally by the urogenital region; the other electrode was placed dorsally, at the nape of the neck. To record heart rate in the 75-day-old subjects, a more chronic electrode was implanted approximately $24 \mathrm{~h}$ prior to testing. This electrode consisted of a Winchester subminiature connector with two $7-\mathrm{cm}$ Teflon-coated leads. Implanting this electrode is a very simple and brief procedure (for more details on the electrode and surgery procedures, see Richardson, Siegel, \& B. A. Campbell, 1988b). The electrodes, and attached leads (32-ga Teflon-coated wire supplied by Alpha), did not appear to interfere appreciably with the subjects' behavior.

Cardiac potentials obtained through these electrodes were amplified by a Grass preamplifier (Model P15). The R-spike activated a Schmitt trigger (Coulbourn Instruments); its output was detected by a microcomputer that measured the interbeat interval (IBI) to the nearest millisecond. The microcomputer controlled all timing sequences and all data collection. The cardiac signal was continuously displayed on a Hitachi oscilloscope (Model V-212).

Treatment of cardiac data. IBIs were measured, to the nearest millisecond, for all subjects during a 5 -sec prestimulus period and during the $10-\mathrm{sec}$ stimulus period on all trials. The IBI data were converted to a beats-per-minute measure for analysis. To measure the cardiac $\mathrm{OR}$, the mean heart rate during the prestimulus period was subtracted from the mean heart rate during the stimulus period. A cardiac $O R$ is indicated by lower heart rates during the stimulus period (resulting in a negative difference score).

Behavioral OR. Earlier work from this laboratory (Siegel et al., 1987) examined the cardiac and behavioral OR to novel auditory, visual, and olfactory stimuli. Although heart rate decreased in response to each of these stimuli, a modality-specific behavioral response was observed. Of importance here is that Siegel et al. (1987) found the behavioral OR to a novel auditory stimulus to be a discrete, ballistic head jerk to one side. This behavioral OR was found to most reliably occur on Trial 1 and to habituate very rapidly thereafter. This behavioral response to novel auditory stimuli has been reported in numerous other studies (e.g., Richardson et al., 1988a; Saiers, Richardson, \& B. A. Campbell, 1989, 1990). In this experiment, each subject's behavioral response was recorded on Trials 1, 5, and 10.

Procedure. On each of the two test days, separated by $24 \mathrm{~h}$, all animals received 10 presentations of a novel auditory stimulus. A 60-sec interval separated successive stimulus presentations. A 15min adaptation period preceded the first stimulus presentation on each day. If an animal was grooming following the adaptation period, the initial stimulus presentation was delayed until this behavior was terminated.

\section{Results}

In all cases where repeated trials were included as a factor in an ANOVA, the Greenhouse-Geisser method was used to control for any potential inflation in the actual probability values. To assess retention of habituation, each animal's response on the first trial of the second session was compared with its response on the last trial of the first session. If forgetting occurs over the 24-h retention interval, then a larger response should occur on the second day than was seen at the end of the habituation session.

Heart-rate OR. The animals of all three ages initially exhibited a substantial decrease in heart rate to the novel auditory stimulus. With repeated presentations of the stimulus, the magnitude of this response decreased to the point where no reliable response was seen on the final trials. These data are presented in the left panel of Figure 1 . When the animals were reexposed to the auditory stimulus $24 \mathrm{~h}$ later, only the 16-day-olds exhibited a response. The 30- and 75-day-olds, on the other hand, exhibited retention of habituation. That is, they did not respond to the previously habituated stimulus following the 24-h retention interval. These data are depicted in the right panel of Figure 1.

Statistical analysis of the heart-rate data confirmed these interpretations. A 3 (age) $\times 10$ (trial) ANOVA of the change in heart rate on the first day of stimulus exposure resulted in a significant effect of trial $[F(9,243)=11.38$, $p<.01]$. The effect of age and the age $\times$ trial interaction were not significant [largest $F(2,27)=1.6, p>.10$ ]

To assess retention of habituation, separate ANOVAs were conducted at each age, comparing the cardiac OR

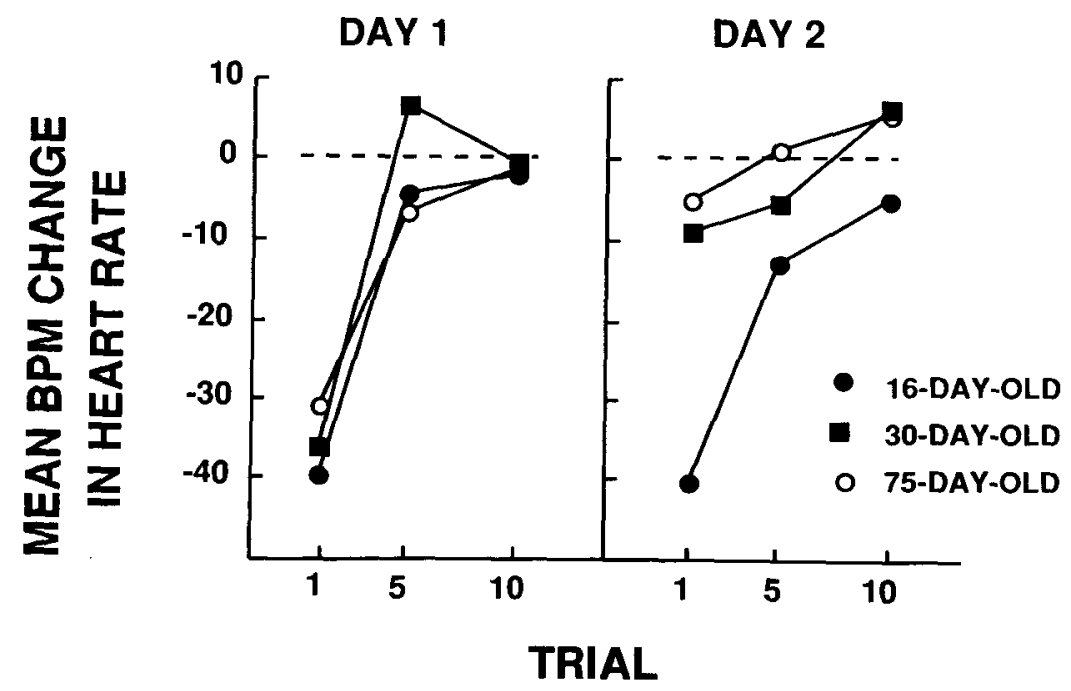

Figure 1. Habituation of the cardiac orienting response to a novel auditory stimulus in 16-, 30-, and 75-day-old rats (left panel); retention of habituation following a 24-h interval (right panel). 

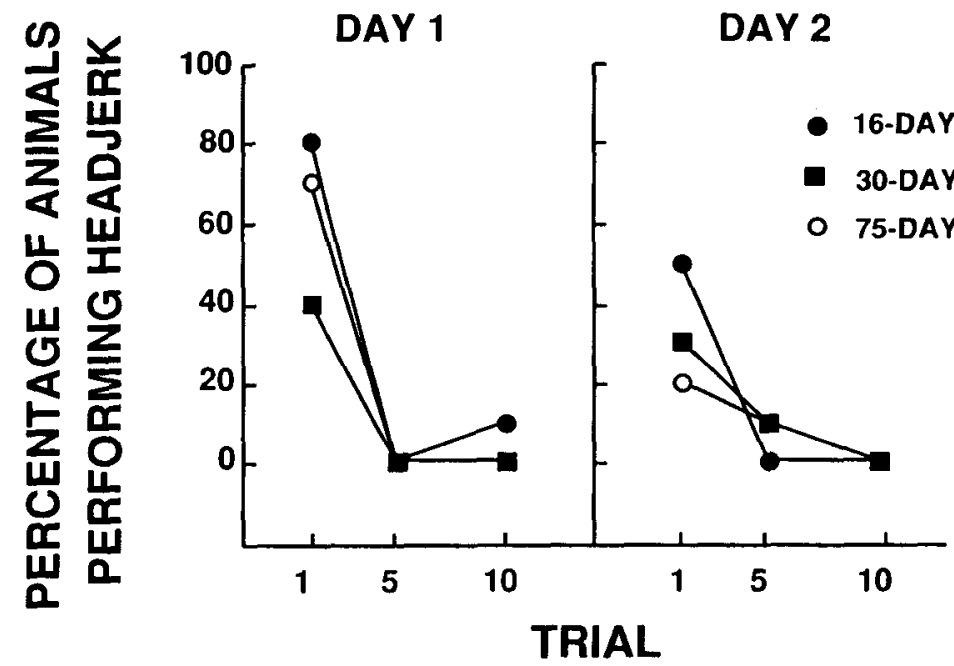

Figure 2. Habituation of the behavioral orienting response to a novel auditory stimulus 16-, 30-, and 75-day-old rats (left panel); retention of habituation following a 24-h interval (right panel).

on the first trial of the second session with the last trial of the first session. The only significant effect found with these tests was for the 16-day-old subjects $[F(1,9)=21.31$, $p<.01]$. The ANOVAs for the two older groups both resulted in $F_{\mathrm{S}}<1.0$. The data presented in Figure 1 clearly indicate that the 16-day-old subjects responded to the auditory stimulus exactly the same way on each day. That is, they treated it as a novel stimulus both times. The 30- and 75-day-olds, however, responded much less on the second day than they did on the first, indicating that they remembered their prior exposure to the stimulus.

Although the 16-day-old subjects initially responded to the previously habituated stimulus, perhaps a "savings" score would reveal some retention in these subjects. A sensitive index of any residual memory would be to compare rates of habituation during each of the two exposure sessions. A $2 \times 10$ (days $\times$ trials) ANOVA on the cardiac OR data of the 16-day-olds resulted in a significant effect of trial $[F(9,81)=8.36, p<.01]$, but no effect of day or interaction of day $\times$ trial (largest $F=1.0$ ). Even by this measure, the 16-day-olds failed to exhibit any evidence of retention of habituation of the OR over a 24-h interval.

Behavioral OR. Although the behavioral data are not nearly as striking as the cardiac data, the same pattern of results was obtained with both measures. On the first test trial, nearly $80 \%$ of the animals at all three ages exhibited a behavioral OR. On the subsequent trials, virtually no responding was seen at any age. Habituation of the behavioral component of the OR to a novel auditory stimulus was significant at all ages [smallest $\chi^{2}(2)=9.25$, $p<.01]$. These data are presented in Figure 2 .

To examine any age differences in retention of habituation, Fisher exact probability tests were performed, at each age, comparing the behavioral response on the last trial on Day 1 and the first trial of Day 2 . The only test that approached significance was that for the 16-day-olds $(p=.065)$. Neither the 30-day-old subjects $(p=.105)$ nor the 75-day-olds $(p=.236)$ exhibited any evidence of forgetting over the 24-h interval.

Basal heart rate. Basal heart rates (obtained during the 5-sec period preceding each stimulus presentation) varied as a function of age: the 16-day-olds had the highest heart rates and the 75-day-olds had the lowest. Mean heart rates (beats-per-minute) on Trials 1, 5, and 10 on each test day are presented in Table 1.

The basal heart rates reported in this study are comparable to those reported in previous studies from this laboratory, as well as work by other investigators. Obviously, these age-related differences in basal heart rate had little, if any, impact on the occurrence or nonoccurrence of the cardiac OR. Analysis of the basal heart-rate data yielded a significant effect of age on each of the two exposure days [smallest $F(2,27)=32.04, p<.01$ ]. Additional ANOVAs were done to examine any potential effects of trial on basal heart rates. Out of the six possible comparisons, only one was significant: the 16-day-old subjects exhibited a significant effect of trial on the second exposure day $[F(9,81)=3.88, p<.05]$. These subjects initially exhibited a slight decrease, followed by a

Table 1

Basal Heart Rates on Each of Two Successive Days for Rats 16, 30, and 75 Days of Age

\begin{tabular}{cccccccc}
\hline & \multicolumn{3}{c}{ Day 1 } & & \multicolumn{3}{c}{ Day 2 } \\
\cline { 2 - 4 } \cline { 6 - 7 } Rats & \multicolumn{3}{c}{ Trial } & & & \multicolumn{3}{c}{ Trial } \\
\hline 16-day-olds & 494 & 5 & 10 & & 1 & 5 & 10 \\
30-day-olds & 463 & 463 & 450 & & 485 & 482 & 506 \\
75-day-olds & 384 & 381 & 369 & & 396 & 369 & 426 \\
\hline
\end{tabular}


more pronounced increase, in basal heart rate over the 10 stimulus presentations. These fluctuations in basal heart rate on the second test day in the 16-day-old subjects had no effect on the reported age-related retention differences: the primary data used from the second day involved only the first trial.

\section{Discussion}

Presentation of a novel auditory stimulus elicited the OR in 16-, 30-, and 75-day-old rats. With repeated exposures, the OR to this stimulus habituated. There were no age differences in rate of habituation of this response. There were, however, marked developmental differences in retention of habituation. Following a 24-h retention interval, the 16-day-old rats exhibited significant forgetting of habituation. The 30- and 75-day-old subjects, however, did not appear to forget over this interval. The older subjects exhibited substantial retention of habituation over the 24-h interval. These results are in agreement with the numerous studies showing more rapid forgetting of associative learning in the preweanling rat than in older animals (for review, see Spear, 1979).

The differences in basal heart rate observed in Experiment 1 were comparable to those reported in other studies with animals ranging from 16 to 75 days of age (Richardson et al., 1988a, 1988b). These differences in basal heart rate did not contribute to the observed age effects on retention. The differences in retention were revealed by statistical analyses done within an age. Furthermore, the same pattern of results seen with the cardiac OR was observed with the behavioral component of the OR. That is, the 16-day-olds forgot overnight, whereas the 30 - and 75-dayolds remembered.

\section{EXPERIMENT 2}

The results of Experiment 1 provide clear evidence of an effect of age on retention of habituation. Although the 30- and 75-day-old rats exhibited retention of habituation overnight, the 16-day-old rats did not. To determine whether the preweanling rat was able to retain habituation over any interval, Experiment 2 was conducted. In this experiment, the retention function of 16-day-old rats over a 24-h period was examined in more detail. Separate groups of animals were tested $20 \mathrm{~min}$ to $24 \mathrm{~h}$ after their initial exposure to the auditory stimulus in order to determine the retention of habituation function of 16-dayold rats.

\section{Method}

Subjects. Forty experimentally naive 16-day-old rats obtained from the same source as those in Experiment 1 were used as subjects. All housing and maintenance procedures were the same as in Experiment 1.

Procedure. All animals received 10 stimulus presentations using the same procedures as in Experiment 1, with one exception-the retention interval. Separate groups of animals $(n s=10)$ received their second series of exposures to the auditory stimulus 20,60, 240 , or $1,440 \mathrm{~min}$ following the first.

\section{Results}

Heart-rate OR. A marked decrease in heart rate was elicited by the novel auditory stimulus when it was first presented. With repeated stimulus presentations, this response habituated. These data are depicted in the left panel of Figure 3. The animals showed a systematic decrease in retention of habituation as a function of time between the two test sessions. The subjects in the 1,440min group exhibited complete forgetting, whereas those tested after $4 \mathrm{~h}$ exhibited only partial forgetting. The subjects tested after either 20 or 60 min exhibited very little, if any, forgetting. These data are presented in the right panel of Figure 3.

Analysis of the cardiac OR during the initial series of stimulus presentations failed to indicate any differences between the subsequently formed groups $(F<1.0)$. These data were therefore collapsed (see Figure 3). A significant effect of trial was seen for the collapsed data $[F(9,324)=28.13, p<.01]$.

To examine retention of habituation, separate ANOVAs were done at each interval, comparing the response on the last trial in the initial series of stimulus exposures (at the end of habituation) with the response to the first stimulus exposure during the second session. These tests yielded significant results for the animals tested at the 240-min interval $[F(1,9)=8.43, p<.01]$ and the 1,440 -min interval $[F(1,9)=18.76, p<.01]$. The subjects tested after either a 20 - or a 60 -min interval did not exhibit a larger response on the test trial than they did on the last habituation trial [largest $F(1,9)=1.11, p>.30]$.

Behavioral OR. The same pattern of results obtained with the cardiac OR was also seen with the behavioral component of the OR. That is, the novel auditory stimulus initially elicited the behavioral response but, with repeated stimulus presentations, habituation of this response occurred. Furthermore, the animals tested after either of the two shorter intervals exhibited retention, whereas those tested after either of the two longer intervals exhibited forgetting. The percentages of animals exhibiting the head-jerk response on Habituation Trials 1, 5 , and 10 were, respectively, 65,2 , and 0 . On the first test trial following a 20-, 120-, 240-, or 1,440-min retention interval, the percentages of animals exhibiting this response were $0,10,50$, and 40 , respectively.

For the initial series of stimulus presentations, all of the data were collapsed into a single group. Analysis of these data yielded a significant effect of trial $\left[\chi^{2}(2)=62.21\right.$, $p<.01]$. Fisher exact tests were then done, for each retention interval, comparing the behavioral response on the last trial of the habituation series and the first trial of the test series. These tests indicated a significant recovery of the habituated behavior only for the subjects tested after either $240 \mathrm{~min}(p=.016)$ or $1,440 \mathrm{~min}(p<.043)$.

Basal heart rate. Since all groups in this experiment were the same age, no group effects on basal heart rate would be expected. None were found. The mean heart rates for the animals in this experiment were comparable to those reported for the 16-day-olds in Experiment 1. Of 


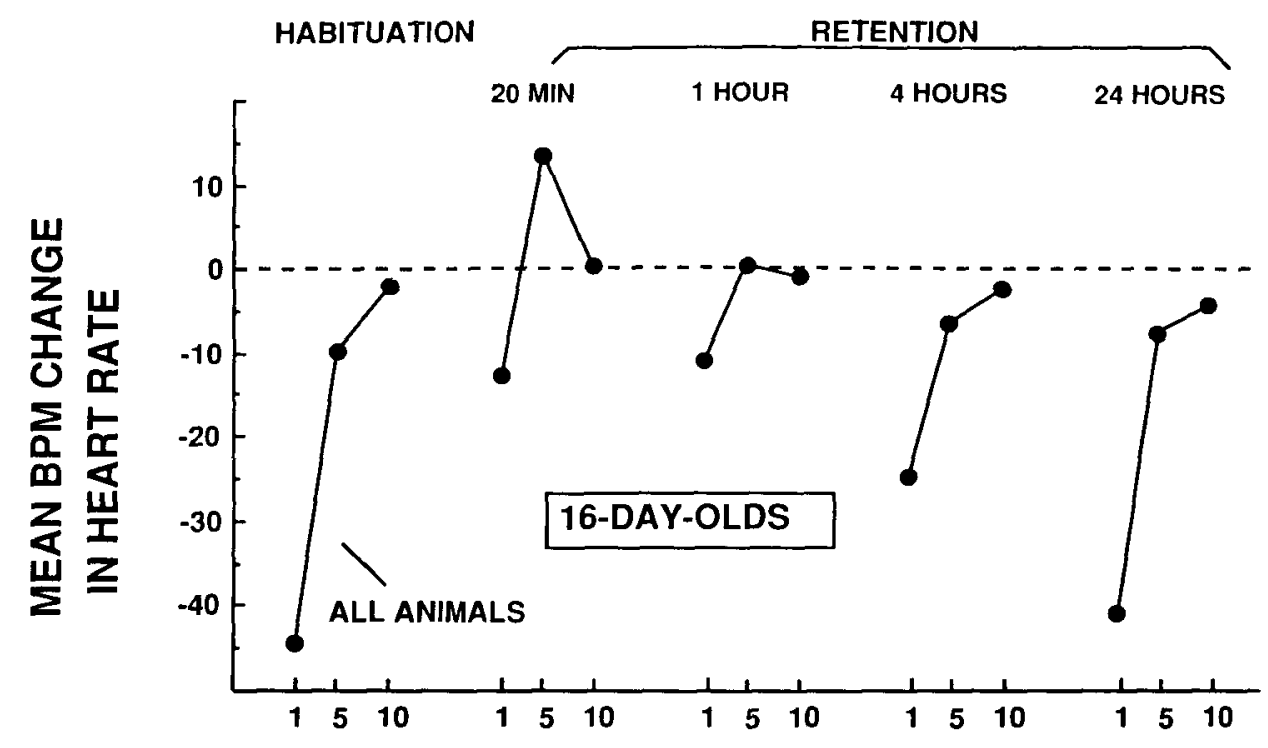

TRIAL

Figure 3. Habituation of the cardiac orienting response to a novel auditory stimulus in 16-day-old rats (left panel); retention of habituation following a 20-, 60-, 240-, or 1,440-min interval (right panel).

the eight ANOVAs conducted to examine the effect of trial on basal heart rate, only one was significant: the animals tested after a 240 -min interval exhibited an effect of trial during their initial exposure session $[F(1,9)=2.97$, $p<.04]$. These animals exhibited a slight reduction in heart rate during the initial 10 trials.

\section{Discussion}

The results of Experiment 2 replicate and extend those of Experiment 1. As in Experiment 1, 16-day-old rats exhibited complete forgetting of habituation over a 24-h interval. Furthermore, testing the animals after only a 4-h interval yielded a similar result. The animals responded to a previously habituated stimulus as if it were novel only $4 \mathrm{~h}$ after the initial habituation series. In contrast, the animals tested either 20 or $60 \mathrm{~min}$ after 10 exposures to a novel auditory stimulus failed to respond when that stimulus was presented again, demonstrating retention of habituation. This pattern of results was found with both a behavioral and an autonomic component of the OR. The results of Experiment 2 demonstrate that preweanling rats retain habituation of the OR for a period of time, albeit a short one.

\section{EXPERIMENT 3}

Experiment 2 determined the retention function for preweanling rats in the present paradigm. In Experiment 3, the retention function of young adult rats for this task was examined. Given that young adult rats exhibited good retention after $24 \mathrm{~h}$ in Experiment 1, the animals in the present experiment were tested 1,3 , or 7 days following their initial exposure to a novel auditory stimulus.

\section{Method}

Subjects. Thirty experimentally naive male rats approximately 75 days of age were used. Housing and maintenance were the same as in the earlier experiments.

Procedure. All procedures were the same as in Experiment 1, with one exception-the retention interval. Independent groups of animals $(n s=10)$ were tested for retention of habituation following a 1-, 3-, or 7-day interval in this experiment. As in the preceding experiments, the retention test consisted of an additional 10 stimulus presentations.

\section{Results}

Heart-rate OR. Presentation of the novel auditory stimulus elicited a marked decrease in heart rate, which habituated with repeated stimulus presentation. As was found with the adult subjects in Experiment 1, the animals in Experiment 3 exhibited retention of habituation over a 24-h interval. Furthermore, in the present experiment, independent groups of animals tested after either a 3- or a 7-day interval also exhibited retention of habituation. These data are shown in Figure 4.

During the first series of stimulus presentations, the three subsequently formed groups did not differ in terms of the cardiac OR $(F<1.0)$. Therefore, these data were collapsed into a single group. Analysis of the collapsed data yielded a significant effect of trial $[F(9,243)=11.14$, $p<.01]$.

To examine retention of habituation, the response on the first trial in the second session was compared with 


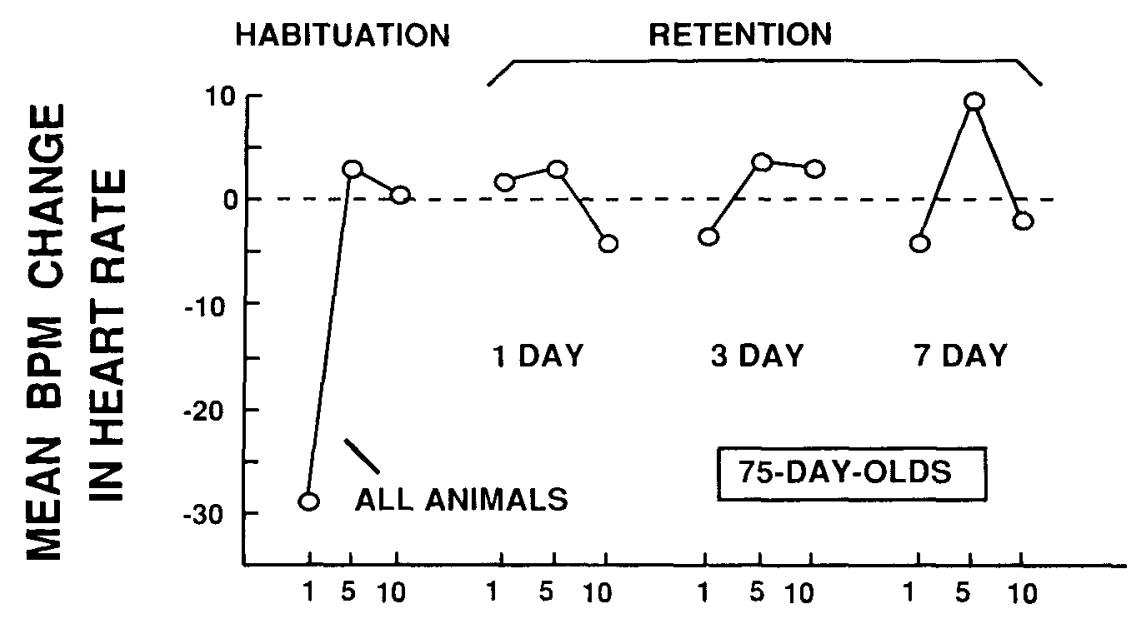

TRIAL

Figure 4. Habituation of the cardiac orienting response to a novel auditory stimulus in 75-dayold rats (left panel); retention of habituation following a 1-, 3-, or 7-day interval (right panel).

the response on the last trial of the first session. These tests were not significant for the animals tested after a 1 -, 3-, or 7-day retention interval [largest $F(1,9)=1.78$, $p>.10]$.

Behavioral OR. The same pattern of results reported for the cardiac OR was obtained with the behavioral OR, with the adult animals exhibiting retention of habituation for at least 7 days. The percentage of animals exhibiting the head-jerk response on Habituation Trials 1, 5, and 10 were, respectively, 57, 3, and 0 . Following a 1-, 3-, or 7 -day retention interval, only $20 \%-30 \%$ of the animals exhibited this response on the first test trial.

Analysis of the behavioral OR during the first exposure session, collapsed across the three subsequently formed groups, resulted in a significant effect of trial $\left[\chi^{2}(2)=\right.$ $37.92, p<.01]$. Presentation of the auditory stimulus elicited the head-jerk response in most of the animals initially, but this behavior rapidly habituated. Fisher exact tests, comparing the behavioral response on the last trial of the first session with the first trial of the second session, were done at each retention interval. None of these tests were significant (all $p \mathrm{~s}>.10$ ).

Basal heart rate. Basal heart rates did not differ as a function of retention interval or trial (all $p s>.10$ ). Mean basal heart rates in Experiment 3 varied from 364 to 399 beats per minute, very comparable to what was reported for 75-day-old rats in Experiment 1.

\section{Discussion}

The results of Experiment 3 replicate and extend those of Experiment 1. As in Experiment 1, the young adult rats exhibited retention of habituation over a 24-h retention interval. Furthermore, testing the animals after even longer intervals did not disrupt the excellent retention exhibited by the subjects this age. The animals tested after either a 3- or a 7-day interval exhibited just as strong retention of habituation as did the animals tested after a 1-day interval. This pattern of results was observed with both an autonomic and a behavioral component of the OR. These results stand in marked contrast to those reported for the preweanling rat in Experiment 2, where retention of habituation was not observed after a $4-h$ interval.

\section{GENERAL DISCUSSION}

One of the most striking results of the present research is the finding that adult rats exposed to only 10 presentations of a novel stimulus, one which initially elicited a large and reliable OR, show neither the cardiac nor the behavioral component of that reaction to the same stimulus following a 7-day retention interval. If we assume, as do most of those working on attentional processes in the context of human psychophysiology, that the OR is not elicited when a stimulus matches a preexisting representation in the central nervous system, then it is clear that long-lasting, nonassociative central representations of auditory stimuli can be rapidly established in the adult rat. Moreover, these central representations (memory) can persist undiminished for substantial periods of time.

Equally striking is the rapidity with which the same central representations fade in the preweanling rat. The $O R$ to the novel auditory stimulus used in this study habituated at the same rate in preweanling and adult rats, implying that the central representation of that stimulus was of comparable strength at both ages. Yet, that representation appears to persist for only a few hours in the young rat. When these results are considered in the broader context of the ontogeny of associative and nonassociative learning, it is evident that both nonassociative and associative learning follow comparable developmental sequences: 
Early in development both types of learning are forgotten much more rapidly than in adulthood. Some have suggested that the more rapid rate of forgetting in young animals may be due to maturation of the central nervous system and/or alterations in perception due to growth during the retention interval (B. A. Campbell \& Spear, 1972). Given that the infant animals began to exhibit forgetting of habituation after only $4 \mathrm{~h}$, the results of the present study are not amenable to such an interpretation. This same point was made by Miller and Spear (1989) in a recent study on Pavlovian conditioning. Thus, rapid forgetting in infants, at least in some situations, must be due to some mechanism other than CNS or physical maturation during the retention interval.

Although direct comparisons between associative and nonassociative learning were not made in the present study, the results suggest that nonassociative learning emerges before associative learning. The basis for this conclusion can be most clearly documented for the autonomic measure used in the present study: heart rate. Between 16 and 75 days of age, there were no age differences in rate of habituation of the cardiac OR to a novel auditory stimulus (see Experiment 1). The ability to exhibit nonassociative learning in this preparation obviously emerges quite early during development. It is not until somewhat later in development that the ability to exhibit conditioned changes in heart rate emerges. Previous research in our laboratory has shown that conditioned cardiac responses to an auditory stimulus cannot be established until the rat is 21 days of age (B. A. Campbell \& Ampuero, 1985). Taken together, these results confirm the general principle put forth by many investigators (Harlow, 1959; Piaget \& Inhelder, 1969; Rudy et al., 1984) that simple types of learning emerge earlier in development than do more complex forms. What cannot be established on the basis of our past and current research is whether long-term memory for these two types of learning reaches adult levels of competence at the same or different ages. For that comparison to be made, carefully executed studies documenting the emergence of long-term memory for both types of learning will have to be conducted. If the two types of memory attain adult levels at different ages, it will imply that different structures and/or processes mediate associative and nonassociative memory. If the two forms of memory reach maturity at the same age, they could be mediated either by the same structures or processes or by different mechanisms that emerge in parallel during ontogenesis.

The findings of the present research also have implications for the relationship between strength of original learning and the ontogeny of long-term memory. In many developmental studies, younger subjects take longer or require more trials to acquire the target information than do adults, thereby permitting the possibility that the poorer memory of infants reflects a learning or encoding deficit rather than a memory or retrieval deficit. Furthermore, a recent study suggests that training different-aged subjects to a common criterion may have important effects on mem- ory that are not revealed by performance during learning (Ohr, Fagen, Rovee-Collier, Hayne, \& Vander Linde, 1989; also see Schulenberg, Riccio, \& Sikes, 1971). The results of the present study, however, demonstrate that there are substantial differences in retention of a nonassociative memory as a function of age in the absence of any detectable differences in the rate of original learning. No significant age-related differences in habituation of either the cardiac or the behavioral components of the OR were found in this research; however, the duration of retention was only a few hours in the young rat and at least a week in adults. This outcome strongly supports the view that the rapid forgetting of nonassociative learning in the preweanling animals reflects a memory loss rather than a learning deficit.

The surprisingly long retention of a nonassociative memory in the adult rat reported in this study is congruent with Leaton's research on retention of habituation of the startle response. In an intriguing study, Leaton (1974) found that disruption of drinking by an auditory startle stimulus diminished with repeated stimulus presentations and that retention of this form of nonassociative learning persisted for at least $\mathbf{4 2}$ days in the adult rat. These data, along with those reported in the present study, are strong evidence for the persistence of certain types of nonassociative memory.

Although the results of the present study have been interpreted as revealing a developmental difference in nonassociative memory, it should be noted that these results could potentially be due to age differences in associative memory. The latter possibility arises from Wagner's powerful analysis of memory processes in animals (e.g., Wagner, 1981). From this perspective, associative processes play an important role in long-term habituation. Specifically, it is argued that animals form an association between a stimulus and the context in which it is presented. Subsequent exposure to that context leads to a retrieval-generated representation of the target stimulus in short-term memory. The presence of this representation in short-term memory dampens the animal's response to the actual physical stimulus. In terms of the present research, the observed results could have been due to the fact that the young animals were forgetting the association between the test environment and the eliciting stimulus more rapidly than were the older animals. Therefore, after a retention interval, exposure to the test context was no longer an effective retrieval cue in the young animals.

The data provided by the experiments in this study are not suitable for distinguishing between these alternative accounts. Given that there have been numerous demonstrations of developmental differences in associative memory (see B. A. Campbell \& Spear, 1972), it would be parsimonious to interpret the present results in that way. However, such an interpretation would, most likely, be incorrect. Justification for this judgment comes from a number of sources.

The majority of recent empirical studies of long-term habituation have failed to find any evidence for the type of associative process posited by Wagner's model (for 
review see Mackintosh, 1987). For example, Schaafsma, Packer, and Siddle (1989) examined the effects of changing the environmental context on long-term habituation of the skin conductance response (SCR) in adult humans. The SCR is an autonomic component of the OR, as is the heart-rate response studied in the present study. Subjects in the Schaafsma et al. study exhibited equivalent longterm habituation whether they were tested in the same environmental context in which they were trained or in a different context. In addition, it should be noted that in several preliminary studies using the exact procedure used in the present study, we have also failed to find any evidence of context-specific habituation in either young or adult rats.

Perhaps even more striking is a recent study by Hall and Channel (1985), who examined the behavioral OR to a visual stimulus in adult rats. After this response had habituated, animals were tested in either the same context or a different one. Hall and Channel reported that animals tested in a different context did not exhibit habituation (Experiment 1). This result is exactly what would be predicted from Wagner's model. Importantly, however, Hall and Channel cleverly revealed that the failure to exhibit habituation in a different context was due to the "sensitizing" effects of the different context (Experiment 2). That is, if the two test contexts were equally familiar, then animals failed to exhibit the $O R$ to the visual stimulus in either context. These results strongly suggest that even when changes in context are found to affect longterm habituation, it is due to a process quite different from Wagner's proposed associative process.

Wagner's model of habituation has had, and will continue to have, great heuristic value. There is increasing empirical evidence, however, that long-term habituation may not involve the type of associative process posited by that model. The examples described above are but a few of the recent studies of habituation that have failed to find the type of context effects predicted by Wagner's model. Therefore, the most probable interpretation of the results of the present study would seem to involve developmental differences in nonassociative memory.

The results reported in this study support the view that long-term retention of nonassociative learning increases substantially during development. However, reoccurrence of the OR after increasingly long intervals could reflect some process other than memory loss. In a recent critique of the visual fixation procedure, Rovee-Collier and Hayne (1987) suggested an interesting non-memory-based explanation for the frequently observed age differences in retention of habituation seen in the human infant. These investigators argued that habituation of visual fixation, considered by many to be a behavioral component of the $\mathrm{OR}$, may be a questionable index of memory function in the human infant. They proposed that this procedure is likely to underestimate the developing organism's memory abilities because young animals may be predisposed to attend to any environmental stimulation not recently encountered, even though the CNS representation of that stimulus is intact. From an evolutionary perspective, reaction to stimulus change may be of particular importance to the young organism because it is much more vulnerable to predation than is the adult. To the extent that orienting alerts the animal to potential danger, it may be prudent for the young animal to react to any stimulus that it had not recently encountered as if it were a novel stimulus. Perhaps the old saying "Better safe than sorry" is particularly applicable early in development.

Experimental evidence supporting the view that the orienting response may occur even though the subject has an intact memory representation of the stimulus is provided by a recent study by Hall and Schachtman (1987), in which adult rats were tested for retention of habituation of the behavioral OR to a visual stimulus. Animals were given 10 nonreinforced presentations of a visual stimulus on each of 16 consecutive days. This stimulus initially elicited a behavioral $O R$, approach to a light on the wall of the test chamber, which habituated with repeated exposures. Following a 1-day retention interval, habituation of the approach response to the light was retained, but, after a 16-day interval, the orienting response reappeared. The animals reacted to the visual stimulus as if it were completely novel following the 16day retention interval. Somewhat surprisingly, however, animals given the same number of stimulus exposures exhibited equivalent latent inhibition when they were tested following either interval. The latter result suggests that retention of habituation of the OR may not be a sensitive measure of the animal's memory for nonreinforced exposures to a nonsignal stimulus.

Perhaps a dissociation similar to that reported by Hall and Schachtman (1987) would be observed in the present paradigm with the preweanling rat. Young animals given nonreinforced presentations of a stimulus may orient to that stimulus when it is presented following some interval, but they may still condition more slowly to that stimulus (i.e., exhibit latent inhibition). Regardless of the ultimate interpretation, however, the data reported in this study convincingly demonstrate that the OR to an auditory stimulus habituates to the same asymptote and at the same rate in both infant and adult rats and that the $O R$ reappears after a markedly shorter retention interval in the younger animals. To the extent that habituation and diminution of habituation of any response with the passage of time reflects acquisition and retention of nonassociative learning, the present results show that there is a substantial increase in long-term nonassociative memory during the course of development.

\section{REFERENCES}

Bailey, C. H., Castellucci, V. F., Koester, J., \& Chen, M. (1983) Behavioral changes in aging Aplysia: A model system for studying the cellular basis of age-impaired learning, memory, and arousal. $B e$ havioral \& Neural Biology, 38, 70-81

Campbell, B. A., \& Ampuero, M. X. (1985). Dissociation of autonomic and behavioral components of conditioned fear during development in the rat. Behavioral Neuroscience, 99, 1089-1102. 
Campeell, B. A., \& Campbell, E. H. (1962). Retention and extinction of learned fear in infant and adult rats. Joumal of Comparative \& Physiological Psychology, 55, 1-8.

Camprell, B. A., \& SPEAr, N. E. (1972). Ontogeny of memory. Psychological Review, 79, 215-236.

Campbell, B. A., \& Stehouwer, D. J. (1980). Retention of habituation and sensitization in neonatal rats. Behavioral and Neural Biology, 29, 190-202.

Carew, T. J., Pinsker, H. M., \& Kandel, E. R. (1972). Long-term habituation of a defensive withdrawal reflex in Aplysia. Science, 175, 451-454.

Cohen, L. B., \& Gelber, E. R. (1975). Infant visual memory. In L. Cohen \& P. Salapatek (Eds.), Infant perception from sensation to cognition: Basic visual processes (Vol. 1, pp. 347-403). New York: Academic Press.

Greco, C., Rovee-Collier, C., Hayne, H., Griesler, P., \& EARLEY, L. (1986). Ontogeny of early event memory: I. Forgetting and retrieval by 2 - and 3-month-olds. Infant Behavior \& Development, 9, 441-460.

Groves, P. M., \& Thompson, R. F. (1970). Habituation: A dual-process theory. Psychological Review, 77, 419-450.

Hall, G., \& Channel, S. (1985). Differential effects of contextual change on latent inhibition and on the habituation of an orienting response. Journal of Experimental Psychology: Animal Behavior Processes, 11, 470-481.

Hall, G., \& Schachtman, T. R. (1987). Differential effects of a retention interval on latent inhibition and the habituation of an orienting response. Animal Learning \& Behavior, 15, 76-82.

HARLOW, H. F. (1959). The development of learning in the rhesus monkey. American Scientist, 47, 459-479.

Hill, W. L., Borovsky, D., \& Rovee-Colluer, C. (1988). Continuities in infant memory development. Developmental Psychobiology, 21, 43-62.

LEATON, R. N. (1974). Long-term retention of the habituation of lick suppression and startle response produced by a single auditory stimulus. Journal of Experimental Psychology: Animal Behavior Processes, 2, 248-259.

MACKINTOSH, N. J. (1987). Neurobiology, psychology, and habituation. Behavior Research \& Therapy, 25, 81-97.

Marcus, E. A., Nolen, T. G., Rankin, C. H., \& Carew, T. J. (1988). Behavioral dissociation of dishabituation, sensitization, and inhibition in Aplysia. Science, 241, 210-213.

Miller, J. S., \& SPEAR, N. E. (1989). Ontogenetic differences in shortterm retention of Pavlovian conditioning. Developmental Psychobiology, 22, 377-387.

Ohr, P. S., Fagen, J. W., Rovee-Collier, C., Hayne, H., \& VANDER LINDE, E. (1989). Amount of training and retention by infants. Developmental Psychobiology, 22, 69-80.

Parsons, P. J., FaGan, T., \& SPEAR, N. E. (1973). Short-term retention of habituation in the rat: A developmental study from infancy to old age. Journal of Comparative \& Physiological Psychology, 84, 545-553.

Piaget, J., \& Inhelder, B. (1969). The psychology of the child. New York: Basic Books.

Pinsker, H., Kupferman, I., Castellucci, V., \& Kandel, E. R. (1970). Habituation and dishabituation of the gill-withdrawal reflex in Aplysia. Science, 167, 1740-1742.
RANkIN, C. H., \& CAREW, T. J. (1988). Dishabituation and sensitization emerge as separate processes during development in Aplysia. Journal of Neuroscience, 8, 197-211.

RANkIN, C. H., CAREW, T. J. (1989). Developmental analysis in Aplysia reveals inhibitory as well as facilitatory effects of tail shock. Behavioral Neuroscience, 103, 334-344.

Richardson, R., Siegel, M. A., \& CAMpbell, B. A. (1988a). Unfamiliar environments impair information processing as measured by behavioral and cardiac orienting responses to auditory stimuli in preweanling and adult rats. Developmental Psychobiology, 21, 491-503.

Richardson, R., Siegel, M. A., \& Campbell, B. A. (1988b). Effect of maternal presence on the fear response to an unfamiliar environment as measured by heart rate in rats as a function of age. Developmental Psychobiology, 21, 613-633.

Rovee-Collier, C., \& HAYNe, H. (1987). Reactivation of infant memory: Implication for cognitive development. In H. W. Reese (Ed.), Advances in child development and behavior (Vol. 20). New York: Academic Press.

Rudy, J. W., VoGt, M. B., \& Hyson, R. L. (1984). A developmental analysis of the rat's learned reactions to gustatory and auditory stimulation. In R. Kail \& N. E. Spear (Eds.), Comparative perspectives on the development of memory. Hillsdale, NJ: Erlbaum.

Saiers, J. A., Richardson, R., CAmpbell, B. A. (1989). Pharmacological dissociation of heart rate and somatomotor components of the orienting response. Psychobiology, 17, 418-423.

Saiers, J. A., Richardson, R., \& Camphell, B. A. (1990). Distuption and recovery of the orienting response following shock or context change in preweanling rats. Psychophysiology, 27, 45-56.

Schanfsma, M. F., Packer, J. S., \& Siddle, D. A. T. (1989). The effect of context change on long-term habituation of the skin conductance response to signal and non-signal stimuli in humans. Biological Psychology, 29, 181-191.

Schulengerg, C. J., Riccio, D. C., \& Sikes, E. R. (1971). Acquisition and retention of a passive-avoidance response as a function of age in rats. Journal of Comparative \& Physiological Psychology, 74, 75-83.

Siegel, M. A., Sananes, C. B., Gaddy, J. R., \& Campbell, B. A. (1987). Dissociation of heart rate and somatomotor orienting responses in preweanling rats. Psychobiology, 15, 122-127.

SoKoLOv, E. N. (1963). Higher nervous functions: The orienting reflex. Annual Review of Physiology, 25, 545-580.

SPEAR, N. E. (1979). Memory storage factors in infantile amnesia. In G. Bower (Ed.), The psychology of learning and motivation (Vol. 13, pp. 91-154). New York: Academic Press.

SpEar, N. E., Miller, J. S., \& Jaglio, J. A. (1990). Animal memory and learning. In M. R. Rozensweig \& L. W. Porter (Eds.), Annual review of psychology (Vol. 41, pp. 169-211). Palo Alto: Annual Reviews.

Sullivan, M. W., Rovee-Collier, C. K., TYNes, D. M. (1979). A conditioning analysis of infant long-term memory. Child Development, 50, 152-162.

WAGNER, A. R. (1981). SOP: A model of automatic memory processing in animal behavior. In N. E. Spear \& R. R. Miller (Eds.), Information processing in animals: Memory mechanisms (pp. 5-47). Hillsdale, NJ: Erlbaum.

(Manuscript received August 28, 1990 revision accepted for publication December 3, 1990.) 